\title{
Artery of the Superior Orbital Fissure: An Undescribed Branch from the Pterygopalatine Segment of the Maxillary Artery to the Orbital Apex Connecting with the Anteromedial Branch of the Inferolateral Trunk
}

\author{
H. Kiyosue, S. Tanoue, N. Hongo, Y. Sagara, and H. Mori
}

\begin{abstract}
BACKGROUND AND PURPOSE: Some branches of the internal maxillary artery have anastomoses with the inferolateral trunk that are important as intracranial-extracranial collateral pathways and as dangerous anastomoses for transarterial embolization of these branches. We present here an undescribed branch potentially anastomosing with the anteromedial branch of the inferolateral trunk, which is provisionally named the artery of the superior orbital fissure, defined as an arterial branch from the pterygopalatine segment of the maxillary artery to the orbital apex at the superior orbital fissure.
\end{abstract}

MATERIALS AND METHODS: Two neuroradiologists reviewed 3D and MPR images of the external and/or common carotid artery with particular interest paid to the artery of the superior orbital fissure in 54 patients who underwent 3D angiography with a field of view covering the pterygopalatine fossa and the cavernous sinus. The underlying diseases in these patients were 17 parasellar hypervascular lesions (including 13 cavernous sinus dural arteriovenous fistulas and 4 meningiomas), 18 internal carotid artery stenoses/occlusions, and 19 other diseases.

RESULTS: The artery of the superior orbital fissure was identified in 20 of 54 patients; it arose at the pterygopalatine segment of the maxillary artery, either singly or from a common trunk with the artery of the foramen rotundum, and ran upward to reach the superior orbital fissure. It anastomosed with the anteromedial branch of the inferolateral trunk at the superior orbital fissure with blood flow toward the cavernous sinus $(n=14)$ and/or the ophthalmic artery $(n=2)$. It was more prominent in parasellar hypervascular lesions and internal carotid artery stenoses/occlusions than in other diseases.

CONCLUSIONS: The artery of the superior orbital fissure, a remnant of the anastomotic artery, was often identified, especially in patients with parasellar hypervascular lesions.

ABBREVIATIONS: ILT = inferolateral trunk; PPF = pterygopalatine fossa; SOF $=$ superior orbital fissure

$\mathrm{T}$ he maxillary artery is a major terminal branch of the external carotid artery, which is divided into 3 segments. The first segment originates anterosuperiorly in the deep parotid gland, and the second segment runs anteriorly to reach the pterygomaxillary fissure. It then turns medially to enter the pterygopalatine fossa (PPF) through the pterygomaxillary fissure, becoming the third segment. ${ }^{1}$ The third segment, the

Received November 11, 2014; accepted after revision February 14, 2015

From the Department of Radiology, Oita University Faculty of Medicine, Oita, Japan.

Paper previously presented at: Annual Meeting of the Radiological Society of North America, November 30-December 5, 2014; Chicago, Illinois.

Please address correspondence to Hiro Kiyosue, MD, 1-1 Idaigaoka, Hasama, Yufu City, Oita 879-5963, Japan; e-mail: hkiyosue@oita-u.ac.jp

- Indicates open access to non-subscribers at www.ajnr.org

http://dx.doi.org/10.3174/ajnr.A4331 terminal part of the maxillary artery, runs transversely in the $\mathrm{PPF}$, which is a narrow space bounded by multiple bony structures. The maxillary arterial branches from the third segment run through various fissures, canals, and foramina to supply various tissues and organs of the surrounding areas. ${ }^{1-7}$ The artery of the foramen rotundum is thought to be the sole arterial branch of the third segment of the internal maxillary artery to enter the cavernous sinus through the foramen rotundum. ${ }^{1,5,6}$ It can supply skull base tumors and dural arteriovenous fistulas, and it has a potential anastomosis with the anterolateral branch of the inferolateral trunk (ILT).

There are 2 communication pathways between the PPF and orbita. The uppermost part of the PPF communicates directly with the orbital apex at the superior orbital fissure (SOF), and the PPF communicates anterolaterally with the inferolateral part of the orbita through the inferior orbital fissure (Fig 1). ${ }^{8,9}$ The 


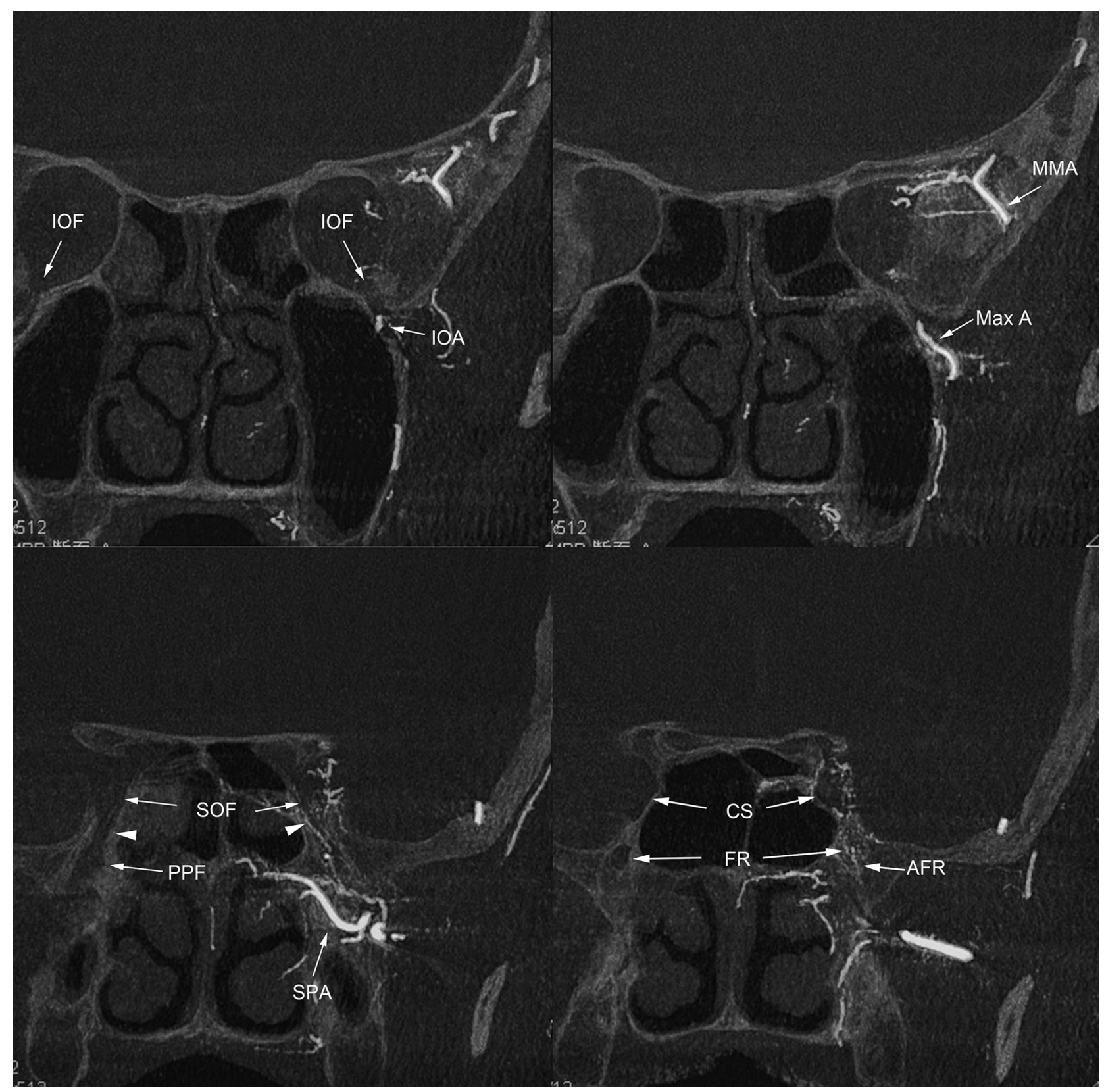

FIG 1. MPR images of rotational angiography in patients with left cavernous sinus dural arteriovenous fistulas. The coronal plane at the level of the inferior orbital fissure (IOF) shows the infraorbital artery (IOA) running through the inferior orbital fissure, through which the pterygopalatine fossa communicates anteriorly with the orbita. The third segment of the maxillary artery (Max A) is located in the pterygopalatine fossa. The coronal plane at the level of the medial part of the PPF shows the pterygopalatine fossa communicating superiorly with the orbital apex (arrowheads) at the SOF. Note a small arterial branch (artery of the SOF) running from the PPF to the SOF through this communication pathway. The PPF communicates medially with the nasal cavity via the sphenopalatine canal containing the sphenopalatine artery (SPA). The PPF communicates posteriorly with the middle cranial fossa via the foramen rotundum (FR), which contains the artery of the foramen rotundum (AFR). The superior orbital fissure continues to the cavernous sinus (CS). MMA indicates middle meningeal artery.

upward communication can be a potential pathway communicating between the PPF and the orbita as well as the middle cranial fossa. Similarly, we found an undescribed branch via the direct communicating pathway between the uppermost part of the PPF and orbital apex, which is provisionally named the artery of the SOF, which originates from the pterygopalatine segment of the maxillary artery and runs upward to reach the orbital apex at the SOF. It frequently anastomoses with the anteromedial branch of the ILT to supply parasellar hypervascular lesions through the
SOF. In this study, we investigated the presence and course of the artery of the SOF in various pathologic conditions by using $3 \mathrm{D}$ rotational angiography.

\section{MATERIALS AND METHODS}

We retrospectively reviewed biplane and 3D rotational angiography of the external carotid artery and/or common carotid artery performed at our institution from June 2010 to February 2014. 
Characteristics of 54 patients

\begin{tabular}{lc}
\hline \multicolumn{1}{c}{ Characteristic } & Patient Data \\
\hline Gender, $n$ & 30 \\
Male & 24 \\
Female & $23-85(67.7)$ \\
Age (range [average]), y & \\
Injected arteries, $n$ & 18 \\
Right ECA & 17 \\
Left ECA & 14 \\
Right CCA & 15 \\
Left CCA & \\
Diseases, $n$ & 13 \\
CSDAVF & 6 \\
Other DAVF & 7 \\
Meningioma & 3 \\
Glioma & 2 \\
Head or neck tumor & 15 \\
ICA stenosis & 3 \\
ICA occlusion & 5 \\
Other disease & \\
\hline
\end{tabular}

Note:-CCA indicates common carotid artery; CSDAVF, cavernous sinus dural arteriovenous fistula; DAVF, dural arteriovenous fistula; ECA indicates external carotid artery.

Datasets of 3D rotational angiography of which the field of view did not cover the entire PPF, orbital apex, or cavernous sinus were excluded. Sixty-four datasets in 54 patients were selected for further evaluation. The characteristics of the 54 patients are summarized in the Table. Patient ages ranged from 23 to 85 years (mean age, 67.7 years), and there were 30 men and 24 women. Underlying diseases included 19 cases of dural arteriovenous fistula at the cavernous sinus $(n=13)$, transverse-sigmoid sinus $(n=2)$, or another location $(n=4) ; 10$ cases of brain tumor (including 7 meningiomas at the paraseller region $[n=4]$ or convexity $[n=$ $3]$ ); 18 cases of internal carotid artery stenosis ( 15 stenoses and 3 occlusions); and 7 other diseases.

Rotational angiography and biplane digital subtraction angiography of the external carotid artery and/or the common carotid artery were performed in all patients by using biplane angiography equipment (Infinix VB; Toshiba Medical, Tokyo, Japan). The rotational angle was $200^{\circ}$, and the rotational speed of the $\mathrm{C}$-arm was $50 \%$ s. The data were acquired in a $512 \times 512$ matrix by using an 8 -inch field-of-view flat panel detector. A nonionic iodinated contrast material (iopamidol [Iopamiron 300; Bayer Health Care, Osaka, Japan]) was injected at a flow rate of $1.5-3.5 \mathrm{~mL} / \mathrm{s}(14-24.5 \mathrm{~mL}$ of total volume) through an automatic injector, and the injection was initiated 1.0-2.0 s before the rotation. The 3D rotational angiography raw data were transferred to a workstation (Ziostation; Ziosoft, Tokyo, Japan), and 3D and MPR images consisting of sections with 0.3 - to $1-\mathrm{mm}$ thickness and a $0.5-\mathrm{mm}$ interval were reconstructed.

All angiographic images and partial MIP and MPR images from the 3D digital angiography were reviewed by 2 experienced neuroradiologists (H.K. and S.T.) to reach consensus on the presence, origin, and course of the artery of the SOF. The artery of the SOF was defined as an arterial branch that originates from the third segment of the maxillary artery and runs superiorly in the pterygopalatine fossa to reach the orbital apex at the SOF. Selective angiography of the feeding arteries of the maxillary artery was also reviewed as a reference when available.

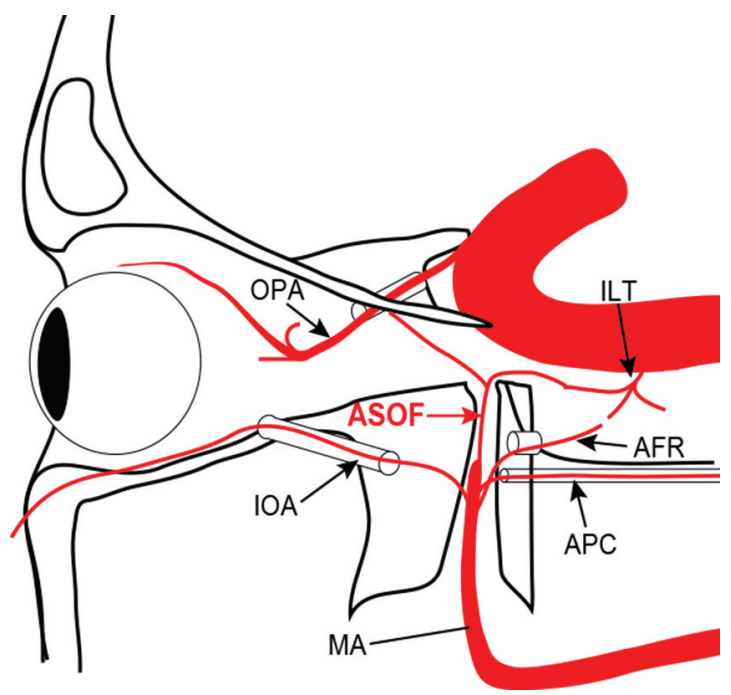

FIG 2. Schematic drawing of the course of the artery of the SOF from the third segment of the maxillary artery (MA). The artery of the SOF (ASOF) runs upward to reach the orbital apex and turns posteriorly to enter the cavernous sinus through the SOF. It has potential anastomosis with the anteromedial branch of the ILT and the ophthalmic artery (OPA). The artery of the foramen rotundum and the artery of the pterygoid canal (APC) run more posteriorly to enter the middle cranial fossa through the foramen rotundum and the foramen lacerum through the pterygoid canal, respectively. The infraorbital artery runs anteriorly to enter the orbital fossa through the inferior orbital fissure.

Each patient was classified into 1 of 3 groups according to the type of underlying disease: 1) parasellar hypervascular disease, including cavernous sinus dural arteriovenous fistulas and parasellar meningiomas, 2) stenosis or occlusive disease of the cervical internal carotid artery, and 3) other disease.

\section{RESULTS}

The artery of the SOF was identified in 20 of 54 patients ( 20 of 64 sides). It originated upward at the pterygopalatine segment of the maxillary artery. It arose by a common trunk, with the artery of the foramen rotundum in 12 patients and independently at a more distal part of the maxillary artery in 8 patients. It ran upward in the upper part of the PPF along its posteromedial wall to reach the orbital apex at the SOF. It anastomosed with the anteromedial branch of the ILT with blood flow toward the cavernous sinus $(n=14)$ and/or the ophthalmic artery $(n=2)$ (Fig 2$)$.

The artery of the SOF was seen as a feeding artery in 11 of 17 patients with parasellar hypervascular lesions ( 9 cavernous sinus dural arteriovenous fistulas and 2 parasellar meningiomas) (Figs 3 and 4) and as a collateral pathway communicating with the anteromedial branch of the inferolateral trunk to the internal carotid artery and/or the ophthalmic artery in 5 of 18 patients with internal carotid artery stenosis/occlusion (Fig 5). Of 19 patients with other diseases, it was observed in 4 patients, and it was very small and could not be traced beyond the SOF in any of the patients.

\section{DISCUSSION}

In human embryos, there are several anastomotic arteries between the maxillary artery and internal carotid artery. These 

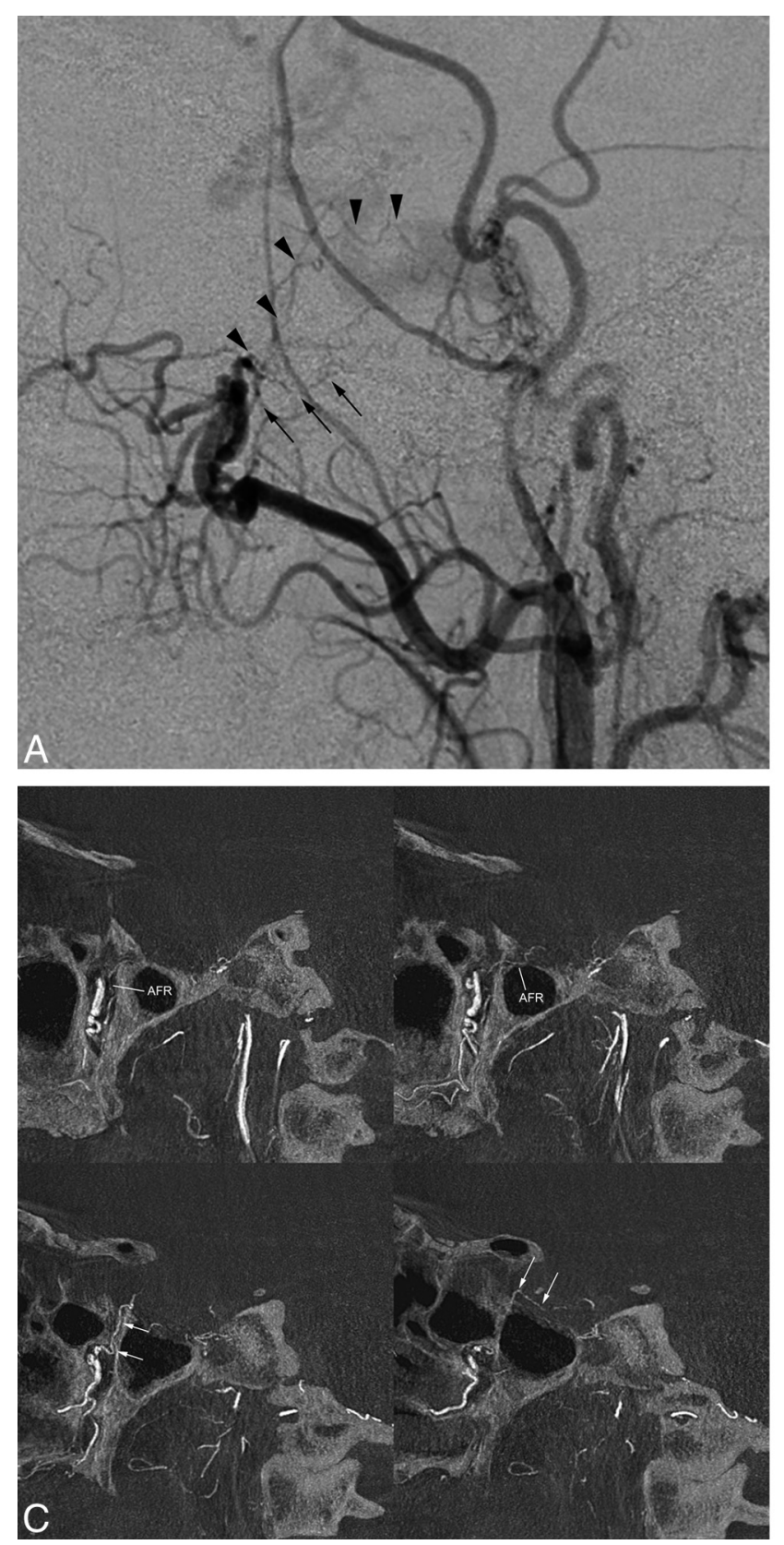

anastomotic arteries regress before birth, and remnants of these anastomotic branches of the internal carotid artery become the branches of the inferolateral trunk. ${ }^{10,11}$ These anastomoses can remain as potential anastomotic pathways communicating between the maxillary artery and the inferolateral trunk, such as the cavernous sinus branch of the middle meningeal artery with the posterolateral branch, posterior branch of the accessory meningeal artery with the posterolateral branch, and the artery of the foramen rotundum with the anterolateral branch of the inferolateral trunk (Fig 6). Among these anastomotic arteries, an anastomotic branch arising from the distal portion of the maxillary artery communicates with the ophthalmic artery and an anastomotic branch of the internal carotid artery, which is a precursor of the anteromedial branch of the inferolateral trunk. The extreme variation of the remnant of anastomoses between the inferolateral trunk

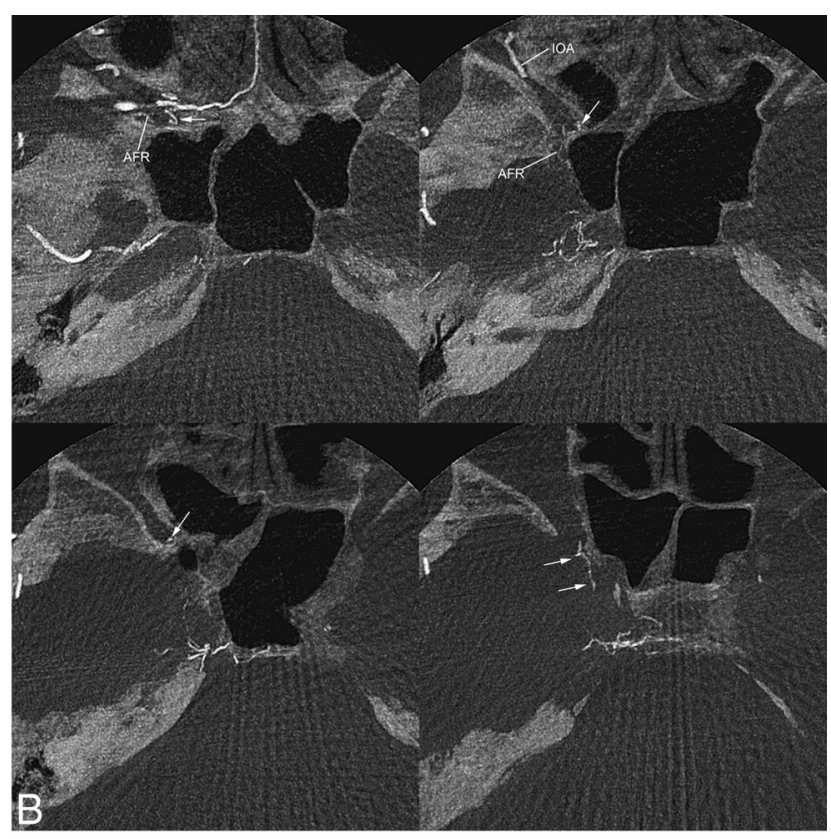

FIG 3. Case of left cavernous sinus dural arteriovenous fistulas supplied by the artery of superior orbital fissure. $A$, The lateral view of the right external carotid angiography shows cavernous sinus dural arteriovenous fistulas fed by multiple feeding arteries, including the artery of the foramen rotundum (arrows) and the artery of the SOF (arrowheads). The artery of the foramen rotundum (arrows) originates posterosuperiorly from the pterygopalatine segment of the maxillary artery and runs posterosuperiorly to the cavernous sinus. The artery of the SOF arises more superiorly from the maxillary artery and runs upward. B, Axial reformatted images of the rotational angiography of the right external carotid artery. The artery of the SOF (arrow) originates at the terminal portion of the maxillary artery just before the sphenopalatine artery. The artery of the foramen rotundum (AFR) originates at the more proximal portion of the pterygopalatine segment of the maxillary artery. The artery of the foramen rotundum runs posteriorly to enter the middle cranial fossa through the foramen rotundum. The artery of the SOF runs upward in the posteromedial portion of the pterygopalatine fossa, and then it runs posteriorly to enter the cavernous sinus through the SOF. The intracranial part of the arterial opacification represents its anastomosis with the anteromedial branch of the inferolateral trunk. Note that the inferior orbital artery (IOA) runs anteriorly to enter the orbital floor through the inferior orbital fissure. C, Sagittal MPR images show that the artery of the foramen rotundum runs posterosuperiorly in the pterygopalatine fossa and enters the middle cranial fossa through the foramen rotundum. The artery of the SOF (arrows) originates independently from the artery of the foramen rotundum and runs upward in the small canal to reach the orbital apex and then turns posteriorly to enter the cavernous sinus through the SOF by anastomosis with the anteromedial branch of the inferolateral trunk.

and the ophthalmic artery is the so-called persistent dorsal ophthalmic artery, coined by Lasjaunias. ${ }^{12}$ Similarly, the remnant of the anastomotic branch from the distal maxillary artery to the ophthalmic artery and the anteromedial branch of the inferolateral trunk would become the artery of the SOF.

In this study, the artery of the SOF anastomosing to the anteromedial branch of the ILT was frequently identified in $65 \%$ of parasellar hypervascular lesions as a feeding artery and $28 \%$ of internal carotid artery steno-occlusive lesions as a collateral pathway. It was observed in other diseases as a very tiny branch, which suggests that the artery of the SOF is difficult to identify and trace unless a pathologic condition increases its blood flow. Therefore, the artery of the SOF has not been described in anatomic studies because of its very small size. Recently, an anatomic study of the pterygopalatine fossa by Oomen et $\mathrm{al}^{13}$ demonstrated a previously undescribed neural branch that communicates with the ophthal- 

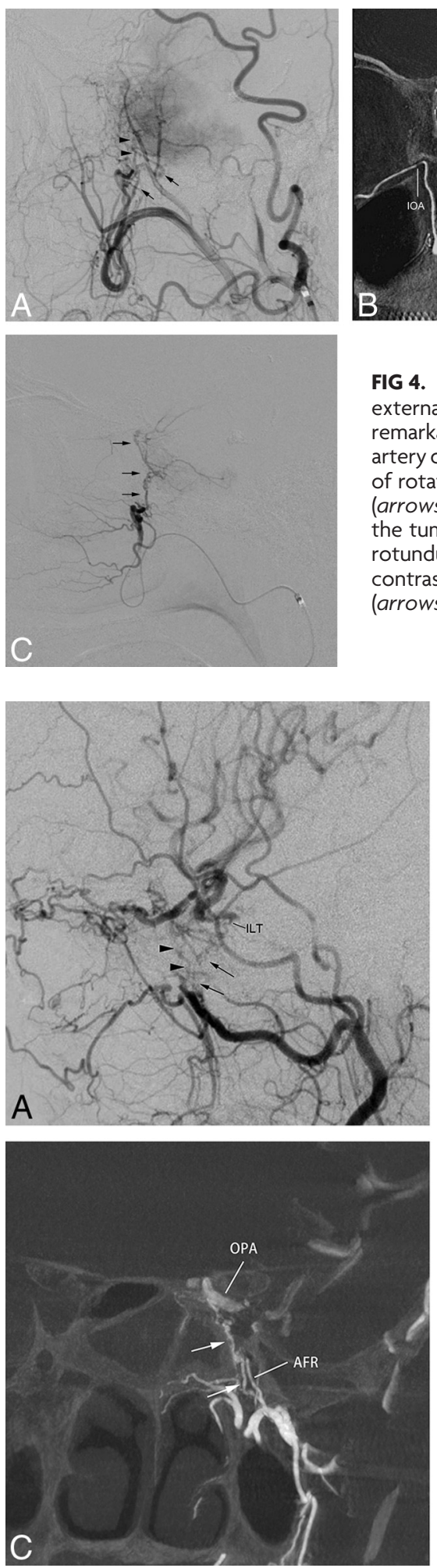

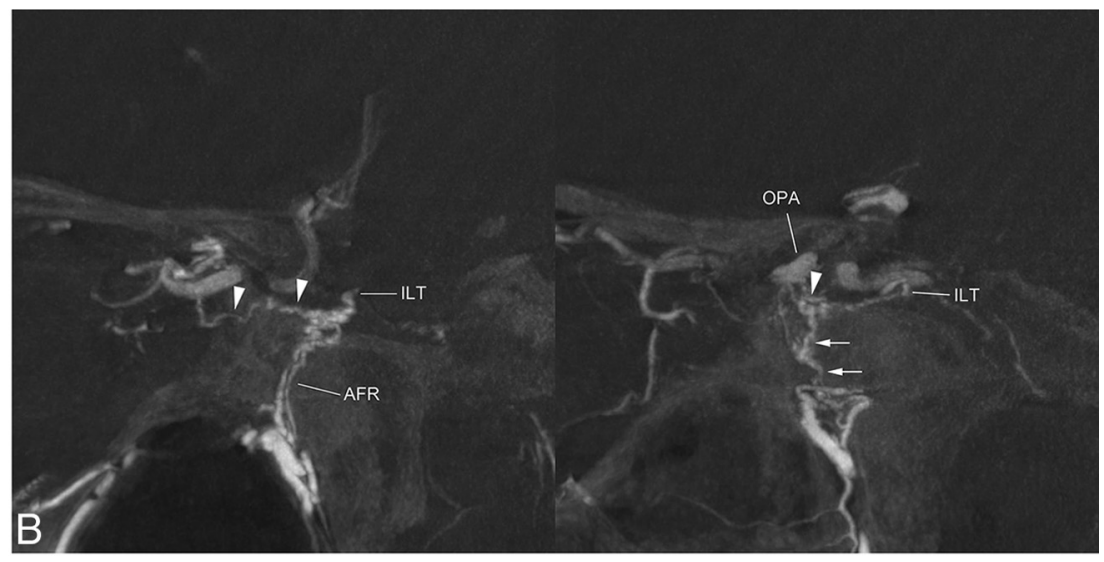

FIG 5. Case of left internal carotid artery occlusion with collateral from the maxillary artery to the internal carotid artery and the ophthalmic artery via the artery of the SOF. $A$, Lateral view of the left common carotid angiography in a patient with occlusion of the cervical internal carotid artery shows multiple collaterals from the external carotid artery to the ophthalmic artery and the ILT of the internal carotid artery. Arrows indicate the artery of the foramen rotundum communicating with the ILT. Arrowheads indicate the artery of the SOF communicating with the ILT and ophthalmic artery. $B$, Sagittal MPR images of rotational angiography of the left common carotid artery show the artery of the foramen rotundum running posterosuperiorly to anastomose with the ILT. The artery of the SOF (arrows) runs upward and connects posteriorly with the ILT and anterosuperiorly with the ophthalmic artery (OPA). Arrowheads indicate the anteromedial branch of the ILT. C, Coronal MPR image of rotational angiography of the left common carotid artery showing that the artery of the SOF (arrows) originates by a common trunk with the artery of the foramen rotundum (AFR). Note an anastomosis between the artery of the SOF and the ophthalmic artery at the orbital apex. mic nerve (V1) and pterygopalatine ganglion. They reported that the nerve was identified in all 5 specimens, and it originated upward from pterygopalatine ganglion and separated into 2 rami entering the orbita anteriorly and cranial cavity posteriorly to join the ophthalmic nerve. The course of this nerve is similar to that of the artery of the SOF in our study. The branches of the maxillary artery often accompany the neural bundle, especially along with branches of the trigeminal nerve. Therefore, the presence of the communicating neural branch between the ophthalmic nerve and the pterygopalatine ganglion supports the presence of the artery of the SOF.

In previous angiographic studies for hypervascular parasellar lesions and collateral pathways between the external carotid artery and internal carotid artery, the artery of the SOF would be ignored or confused with the artery of the foramen rotundum or other maxillary arterial branches because of the lack of recogni- 

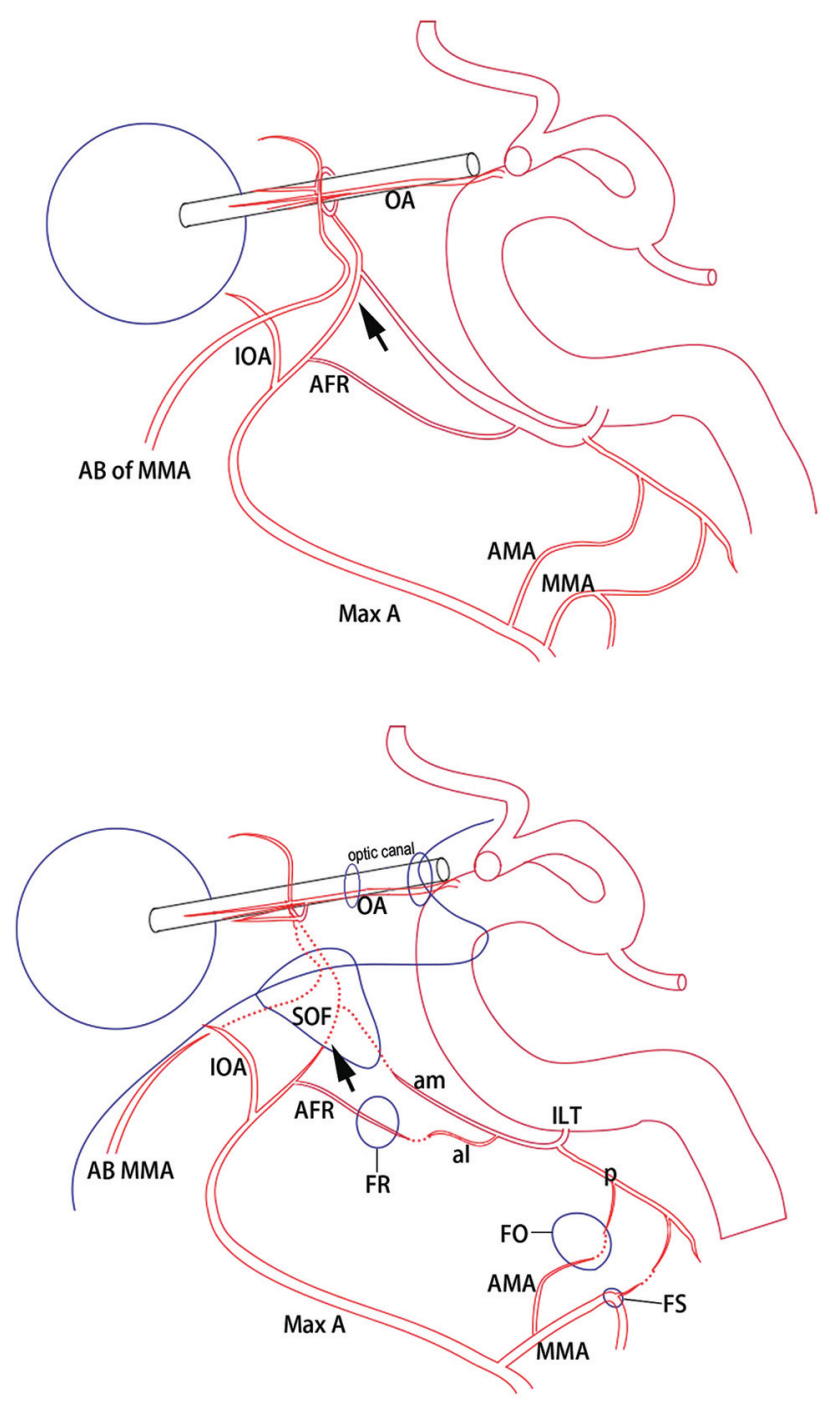

FIG 6. Schematic drawing of the anastomotic arteries between the maxillary artery and internal carotid artery in the fetal period (upper figure) and potential anastomosis in an adult (lower figure). The ophthalmic artery anastomoses with the anterior branch of the middle meningeal artery ( $\mathrm{BB}$ of MMA) and an ophthalmic branch of the maxillary artery (arrow). Several branches arising from the internal carotid artery anastomose with the branches of the maxillary arteries, including the middle meningeal artery (MMA), accessory meningeal artery (AMA), artery of the foramen rotundum (ARF), and the ophthalmic branch of the maxillary artery (arrow) in the fetal period. Anastomotic branches from the internal carotid artery become branches of the ILT. There are several potential anastomoses between the maxillary artery branches and the ILT. The ophthalmic branch of the maxillary artery and its anastomoses in the fetal period becomes the artery of the superior orbital fissure.

tion and knowledge of this artery. Furthermore, it is difficult to evaluate the exact course of the small branch by conventional DSA because of overlapping vessels and a lack of information on the relationship of the branches of the maxillary artery and the complicated bony structure of the orbital apex and PPF. We used MPR and $3 \mathrm{D}$ images reconstructed from a rotational angiography dataset. It has been reported that CT-like reconstructions of 3D rotational angiography are superior to 2D DSA in assessing vascular structures and their relationships with bony structures. ${ }^{14,15}$ After recognition of the discriminative course of the artery of the SOF via 3D rotational angiography, it can be identified easily on the lateral view in conventional DSA. It goes straight upward to reach the level of the orbital apex, and then it turns posteriorly with an approximately $90^{\circ}$ angle to enter the middle fossa by anastomosis with the intracranial portion of the anteromedial branch of the inferolateral trunk. It can also run anterosuperiorly in the orbital fossa to connect to the ophthalmic artery by anastomosis with the orbital portion of the anteromedial branch of the inferolateral trunk. The artery of the foramen rotundum also originates at the pterygopalatine segment of the maxillary artery, but it can be differentiated from the artery of the SOF on DSA because it runs more dorsally up to enter the middle fossa through the foramen rotundum.

Regarding the clinical importance of the artery of the SOF, it can work as a collateral pathway, communicating between the external carotid artery and the internal carotid artery and ophthalmic artery in steno-occlusive diseases of the internal carotid artery. Furthermore, it may be important to evaluate for nasopharyngeal tumors and infections, because some tumors or infectious diseases, such as adenoid cystic carcinoma, can spread along the neurovascular bundle. ${ }^{16}$ Although it is well known that the foramen rotundum is an important canal through which tumors extend from the PPF into the cranial cavity, a tumor can spread from the PPF through the SOF into the cranial cavity along the artery of the SOF.

As mentioned previously, the artery of the SOF frequently works as a feeding artery for parasellar hypervascular lesions, such as meningiomas and dural arteriovenous fistulas, which are often treated by endovascular techniques. Embolization from the artery of the SOF is more dangerous than from other branches of the maxillary artery because it has a potential risk of migration of embolic materials into the internal carotid artery as well as the ophthalmic artery. Despite the apparent communication of the artery of the SOF with the ophthalmic artery, and with the anteromedial branch of the ILT (the embryonic dorsal ophthalmic artery, as termed by Lasjaunias et al), it does not seem that the artery of the SOF serves as a major pathway for collateral reconstitution of the ophthalmic artery. In fact, its existence may have been hitherto overlooked simply because it is so small. However, the possibility now exists, and extra care must be taken. Furthermore, cranial nerve injury of the ophthalmic nerve (V1), which is potentially supplied by the artery of the SOF, is another potential risk of embolization. Therefore, recognition of the artery of the SOF is important for clinical practice.

This study had some limitations. We included a relatively small number of cases, especially for normal circulation in the evaluated area. A low frequency of the presence of the artery of the SOF in patients without hypervascular parasellar lesions may be attributed to the limitations of current $3 \mathrm{D}$ rotational angiography resolution for depicting tiny arteries. Therefore, the exact frequency of the presence of the artery of the SOF is unclear.

\section{CONCLUSIONS}

The artery of the SOF, an undescribed anastomotic artery between the third segment of the maxillary artery and the anteromedial branch of the ILT, was often identified, especially in patients with parasellar hypervascular lesions. A special attention 
should be paid for the presence of the artery of the SOF during transarterial embolization of the branches originating from the third segment of the maxillary artery.

\section{REFERENCES}

1. Djindjian R, Merland JJ. Super-Selective Arteriography of the External Carotid Artery. Berlin: Springer-Verlag; 1978:22-36

2. Daniels DL, Mark LP, Ulmer JL, et al. Osseous anatomy of the pterygopalatine fossa. AJNR Am J Neuroradiol 1998;19:1423-32 Medline

3. Osborn AG. Radiology of the pterygoid plates and pterygopalatine fossa. AJR Am J Roentgenol 1979;132:389-94 CrossRef Medline

4. Kim HS, Kim DI, Chung IH. High-resolution CT of the pterygopalatine fossa and its communications. Neuroradiology 1996;38: S120-26 CrossRef Medline

5. Lasjaunias P, Berenstein A, ter Brugge KG. Clinical Vascular Anatomy and Variations. Berlin: Springer-Verlag; 2001:15-87

6. Tanoue $\mathrm{S}$, Kiyosue $\mathrm{H}$, Mori $\mathrm{H}$, et al. Maxillary artery: functional and imaging anatomy for safe and effective transcatheter treatment. Radiographics 2013;33:e209-24 CrossRef Medline

7. Daniels DL, Rauschning W, Lovas J, et al. Pterygopalatine fossa: computed tomographic studies. Radiology 1983;149:511-16 CrossRef Medline

8. Williams PL, Gray H, Bannister LH. Gray's Anatomy: The Anatomical Basis of Medicine and Surgery. Edinburgh: Churchill Livingstone; 1999
9. Rusu MC, Didilescu AC, Jianu AM, et al. 3D CBCT anatomy of the pterygopalatine fossa. Surg Radiol Anat 2013;35:143-59 CrossRef Medline

10. De La Torre E, Netsky MG. Study of persistent primitive maxillary artery in human fetus: some homologies of cranial arteries in man and dog. Am J Anat 1960;106:185-95 CrossRef

11. Lasjaunias P, Moret J, Mink J. The anatomy of the inferolateral trunk (ILT) of the internal carotid artery. Neuroradiology 1977;13: 215-20 CrossRef Medline

12. Willinsky R, Lasjaunias $P$, Berenstein A. Intracavernous branches of the internal carotid artery (ICA): comprehensive review of their variations. Surg Radiol Anat 1987;9:201-15 CrossRef Medline

13. Oomen KP, Ebbeling M, de Ru JA, et al. A previously undescribed branch of the ganglion. Am J Rhinol Allergy 2011;25:50-53 CrossRef Medline

14. Hiu T, Kitagawa N, Morikawa M, et al. Efficacy of DynaCT digital angiography in the detection of the fistulous point of dural arteriovenous fistulas. AJNR Am J Neuroradiol 2009;30:487-91 CrossRef Medline

15. Kiyosue H, Tanoue S, Okahara M, et al. Angioarchitecture of transverse-sigmoid sinus dural arteriovenous fistulas: evaluation of shunted pouches by multiplanar reformatted images of rotational angiography. AJNR Am J Neuroradiol 2013;34:1612-20 CrossRef Medline

16. Ginsberg LE, DeMonte F. Imaging of perineural tumor spread from palatal carcinoma. AJNR Am J Neuroradiol 1998;19:1417-22 Medline 